\title{
Photoinjector generation of a flat electron beam with transverse emittance ratio of 100
}

\author{
P. Piot, ${ }^{1,2, *}$ Y.-E Sun, ${ }^{3, \dagger}$ and K.-J. Kim ${ }^{3,4}$ \\ ${ }^{1}$ Northern Illinois University, DeKalb, Illinois 60115, USA \\ ${ }^{2}$ Fermi National Accelerator Laboratory, Batavia, Illinois 60510, USA \\ ${ }^{3}$ University of Chicago, Chicago, Illinois 60637, USA \\ ${ }^{4}$ Advanced Photon Source, Argonne National Laboratory, Argonne, Illinois 60439, USA
}

(Received 2 November 2005; published 7 March 2006)

\begin{abstract}
The generation of a flat electron beam directly from a photoinjector is an attractive alternative to the electron damping ring as envisioned for linear colliders. It also has potential applications to light sources such as the generation of ultrashort x-ray pulses or Smith-Purcell free electron lasers. In this paper, we report on the experimental generation of a flat beam with a measured transverse emittance ratio of $100 \pm$ 20 for a bunch charge of $\sim 0.5 \mathrm{nC}$; the smaller measured normalized root-mean-square emittance is $\sim 0.4 \mu \mathrm{m}$ and is limited by the resolution of our experimental setup. The experimental data, obtained at the Fermilab/NICADD Photoinjector Laboratory, are compared with numerical simulations and the expected scaling laws.
\end{abstract}

DOI: 10.1103/PhysRevSTAB.9.031001

PACS numbers: 29.27.-a, 41.75.Fr, 41.85.-p

\section{INTRODUCTION}

Flat electron beams, i.e., beams with large transverse emittance ratios, have been proposed in the context of linear colliders and some novel electron-beam-based light sources. In the case of a linear $e^{+} / e^{-}$collider, a flat beam at the interaction point reduces the luminosity disruption caused by bremsstrahlung [1]. In the case of light sources, such as the LUX project proposed at LBL [2], a flat beam with a smaller emittance of $0.3 \mu \mathrm{m}$ and emittance ratio of 50 is needed to produce x-ray pulses that can be compressed to the order of femtoseconds via standard $\mathrm{x}$-ray pulse compression techniques [3]. Another type of light source recently drawing attention is based on the selfamplification of Smith-Purcell radiation [4]. Given one or two planar metal gratings, a flat beam could enhance the interaction between the electrons and metal grating surface, thus reducing the gain length associated with the Smith-Purcell free-electron-laser mechanism [5-7].

In the proposed International Linear Collider (ILC) the needed flat-beam parameters (emittance ratio of 300) are foreseen to be achieved via radiation cooling in a damping ring [8]. Although the required transverse emittances for the ILC have been demonstrated at the Accelerator Test Facility damping ring of KEK [9], ILC puts stringent requirements on the damping ring design, and the cost of the damping ring is a significant portion of the total collider cost. Therefore alternative ways of producing flat beams directly from an electron source have been explored by several groups [10]. In conjunction with the invention of a linear transformation capable of transforming an incoming flat beam into an angular-momentum-dominated (or " $\mathrm{mag}$ -

\footnotetext{
*Electronic address: piot@nicadd.niu.edu

${ }^{\dagger}$ Electronic address: yinesun@uchicago.edu

Present address: Argonne National Laboratory, Argonne, IL, USA
}

netized") beam [11], a scheme that inverts this transformation was proposed to generate a flat beam directly out of a photoinjector [12]. The method consists of generating a magnetized beam by immersing the photocathode in an axial magnetic field. After acceleration, the beam is transformed into a flat beam using three skew quadrupoles [13]. This has been verified experimentally [14-17], and transverse emittance ratios of 40-50 were reported. Theoretical analysis of the conversion of a magnetized cylindrically symmetric beam into a flat beam has been presented $[18,19]$ and some of the associated limitations explored [20,21]. In the present paper we report on an improvement of the experimental conditions and methods that led to a measured transverse emittance ratio of approximately 100 .

\section{EXPERIMENTAL METHODS}

The flat-beam experiment was carried out at the Fermilab/NICADD ${ }^{1}$ Photoinjector Laboratory (FNPL) [22]; see Fig. 1 for the layout. In brief, electron bunches with variable charge $(Q \leq 20 \mathrm{nC})$ are generated via photoemission from a cesium telluride photocathode located at the back plate of a $1+1 / 2$ cell radio-frequency (rf) cavity operating at $1.3 \mathrm{GHz}$ (the "rf gun"). The beam is then accelerated in a $1.3 \mathrm{GHz}$ superconducting rf cavity (the booster cavity) [23] to approximately $16 \mathrm{MeV}$. The rf gun is surrounded by three solenoidal lenses that are designed to control the beam's transverse emittance. For flat-beam production the first solenoidal lens (L1) is turned off, and the two others (L2 and L3) are tuned to provide the desired magnetic field on the photocathode along with the proper focusing. The beam is thereby produced in the presence of a significant axial magnetic field and has an average angu-

\footnotetext{
${ }^{1}$ NICADD is an acronym for Northern Illinois Center for Accelerator and Detector Development.
} 


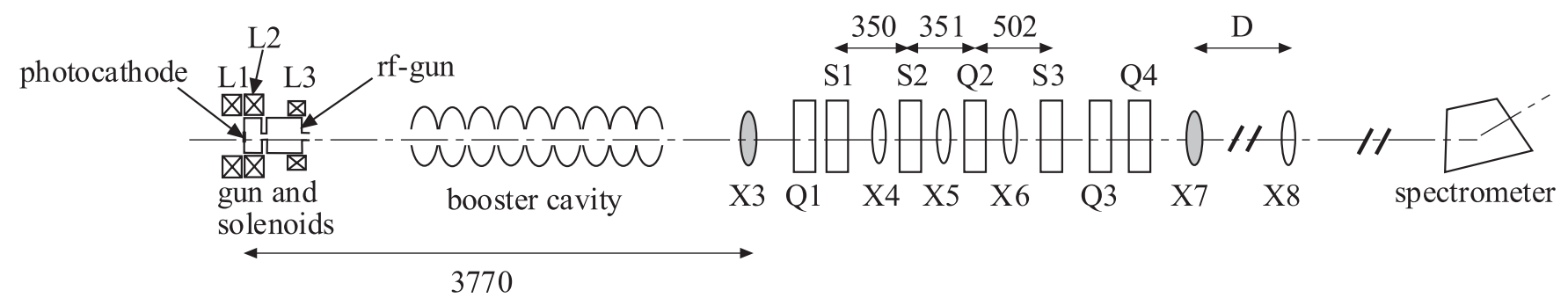

FIG. 1. Overview of the Fermilab/NICADD photoinjector. " $\mathrm{X}$ " refer to diagnostics stations (beam viewers, and/or slit location), "L" to the solenoidal lenses, "Q" to quadrupoles, and "S" to the skew quadrupoles. All distances are in mm, with $D=800$ (or 1850 for the data presented in Fig. 7).

lar momentum given by $\langle L\rangle=e B_{0} \sigma_{c}^{2}$, where $e$ is the electron charge, $B_{0}$ the axial magnetic field on the photocathode surface, and $\sigma_{c}$ the root-mean-square (rms) transverse size of the drive-laser spot on the photocathode. The transformation of the magnetized beam into a flat beam occurs downstream of the booster cavity. Three skew quadrupoles ( $\mathrm{S} 1, \mathrm{~S} 2$, and $\mathrm{S} 3$ in Fig. 1) provide a net torque on the beam thereby removing its initial angular momentum $[24,25]$. The skew quadrupoles main characteristics are: an effective length of $0.102 \mathrm{~m}$ and a maximum field gradient of $\sim 7 \mathrm{~T} / \mathrm{m}$ for a current of $10 \mathrm{~A}$; each quadrupole was calibrated prior to the experiment. The set of skew quadrupoles is henceforth referred to as the round to flat-beam transformer or "transformer" in short. Given the incoming $4 \times 4$ beam covariance matrix $\Sigma_{0}$, the quadrupole strengths are set to provide the proper transport matrix $M$ so that the covariance matrix at the exit of the transformer, $\Sigma=M \Sigma_{0} \tilde{M}$ (where the upper tilde denotes the transpose), is block diagonal. An analytical solution for the quadrupole settings was derived under the thin-lens approximation for the quadrupoles [16]. This solution is used as a starting point for a simplex minimization algorithm that searches the quadrupole settings to minimize the figure-of-merit $\chi^{2}=\Sigma_{13}^{2}+\Sigma_{14}^{2}+\Sigma_{23}^{2}+\Sigma_{24}^{2}$, where $\Sigma_{i j}$ is the $(i j)$ th element of matrix $\Sigma$. Upon proper tuning of the transformer, the expected normalized flat-beam emittances, $\varepsilon_{n}^{ \pm}$, are given by $[18,19]$

$$
\begin{aligned}
\varepsilon_{n}^{ \pm}= & \sqrt{\left(\varepsilon_{n}^{u}\right)^{2}+(\beta \gamma \mathcal{L})^{2}} \\
& \pm(\beta \gamma \mathcal{L}) \stackrel{\beta \gamma \mathcal{L} \gg \varepsilon_{n}^{u}}{\longrightarrow}\left\{\begin{array}{l}
\varepsilon_{n}^{+} \simeq 2 \beta \gamma \mathcal{L}, \\
\varepsilon_{n}^{-} \simeq \frac{\left(\varepsilon_{n}^{u}\right)^{2}}{2 \beta \gamma \mathcal{L}},
\end{array}\right.
\end{aligned}
$$

where $\varepsilon_{n}^{u}=\beta \gamma \varepsilon_{u}$ is the normalized uncorrelated emittance of the magnetized beam prior to the transformer, $\beta=$ $v / c, \gamma$ is the Lorentz factor, $\mathcal{L}=\langle L\rangle / 2 p_{z}$, and $p_{z}$ is the longitudinal momentum. Note that $\varepsilon_{n}^{+} \varepsilon_{n}^{-}=\left(\varepsilon_{n}^{u}\right)^{2}$. A simulation of the beam dynamics along the FNPL beam line is presented in Fig. 2.

The flat-beam emittances are measured using the slit method [26]. A movable single-slit assembly (either vertical or horizontal), located at position X7 (see Fig. 1), is used to sample the beam in one direction. The slit assembly consists of a $\sim 50 \mu \mathrm{m}$ slit made of a $3 \mathrm{~mm}$ thick tungsten block. The beamlet passing through the slit is observed after a drift of distance $D$, at the location X8. Given the measured horizontal beam size at X7, $\sigma_{x}^{X 7}$, and horizontal rms size of the beamlet at X8 when a vertical slit is inserted at $\mathrm{X} 7, \sigma_{x}^{X 8, h}$, the horizontal emittance is then computed as the product $\varepsilon_{n}^{x}=\gamma \sigma_{x}^{X 7} \sigma_{x}^{X 8, h} / D$. Similarly the vertical emittance is measured as $\varepsilon_{n}^{y}=\gamma \sigma_{y}^{X 7} \sigma_{y}^{X 8, v} / D$ where $\sigma_{y}^{X 8, v}$ is the vertical rms size of the beamlet at X8 when a horizontal slit is inserted at X7. The beam viewer at locations $\mathrm{X} 7$ is an optical transition radiation foil, while at $\mathrm{X} 8$ it is a yttrium aluminum garnet screen. The measured rms beam size, $\sigma_{\text {meas }}$, is affected by the resolution of the diagnostics $\sigma_{\text {res }}$ and spurious dispersion $\eta$ introduced, e.g., by steering dipoles required to keep the beam centered along the beam line axis: $\sigma_{\text {meas }}=\sqrt{\sigma^{2}+\sigma_{\text {res }}^{2}+\left(\eta \sigma_{\delta}\right)^{2}}$, where $\sigma_{\delta}$ is the rms fractional momentum spread of the beam. The measurement method used to report emittances in the following discussion was numerically benchmarked [25]. The resolution of the beam size measurement system which includes the optical components and a charged coupled device camera was characterized for various operating points [25]. For all the quoted measurements of transverse beam sizes, we quadratically subtract the smallest measured resolution $\left(\sigma_{\text {res }}=35 \mu \mathrm{m}\right)$. The unavoidable contribution from spurious dispersion (discussed later) results in an overestimated value for the smaller flatbeam emittance. Hence the emittance ratio reported hereafter is underestimated.

For the flat-beam experiment reported in this paper, the nominal operating parameters for the photoinjector are reported in Table I. The rf-gun and booster-cavity settings are kept the same during the experiment while the drivelaser spot size on the photocathode and the solenoid currents are adjusted for the different sets of measurements. Compared to the experiment reported in Refs. [14-16], the experimental conditions were improved: (1) the photocathode drive-laser bunch-to-bunch jitter was reduced, (2) the optical systems used to image the beam spot from the viewers were enhanced to allow single-bunch measure- 

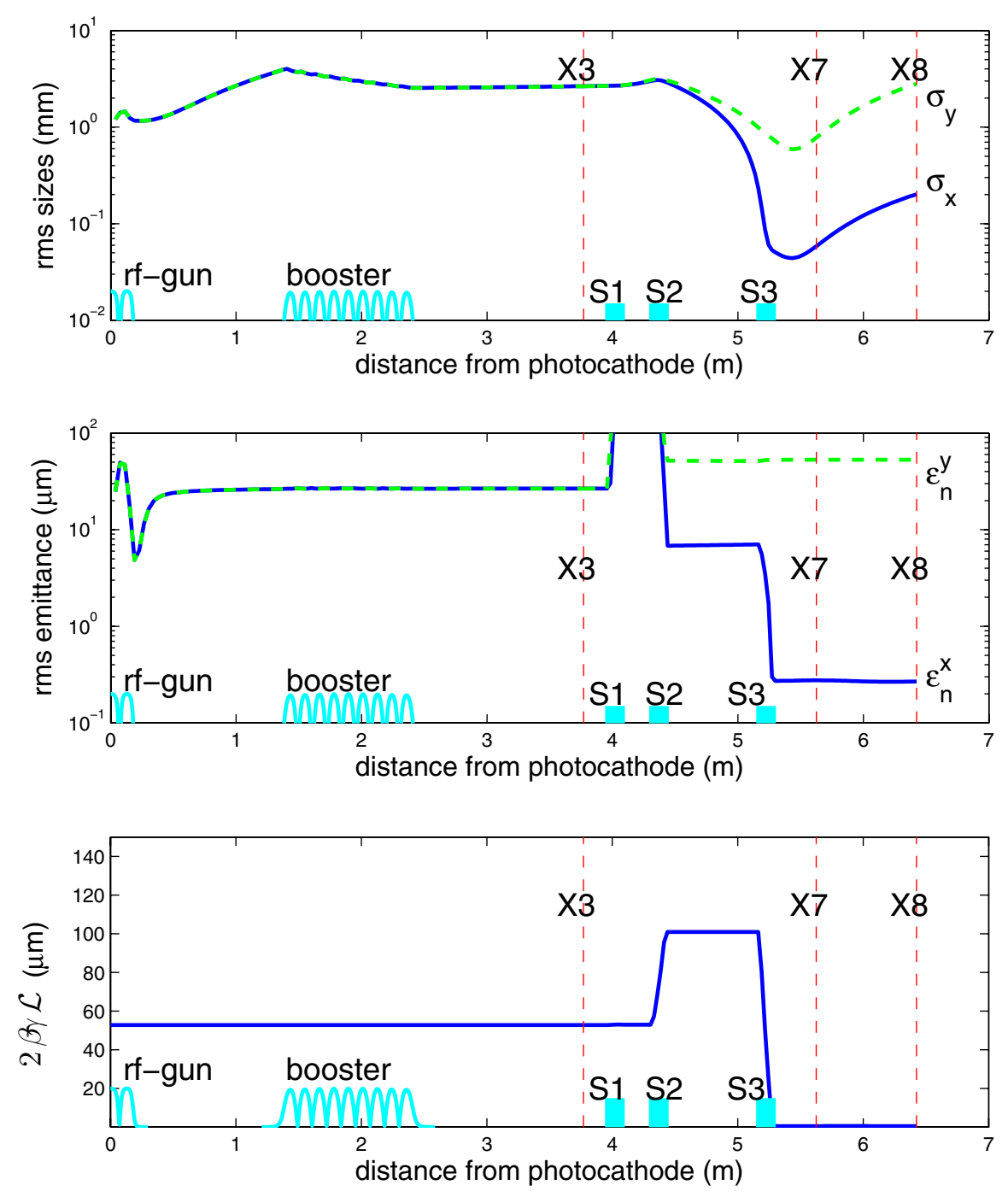

FIG. 2. (Color) Evolution of beam rms transverse sizes (top), normalized emittances (middle), and angular momentum (bottom) along the FNPL beam line from the photocathode up to viewer X8 [the simulation corresponds to the case $\sigma_{c}=0.97 \mathrm{~mm}$ (see text for details)]. The vertical dash lines and the filled boxes, respectively, represent the viewer and skew quadrupole locations. The maximum values for the horizontal and vertical emittances (middle plot) are, respectively, 365 and $417 \mu \mathrm{m}$ (these values are reached between quadrupoles S1 and S2).

ments, with a resolution improved by a factor of 2 , and (3) numerical simulations performed during the experiment provided guidance for tuning the accelerator (e.g. finding the optimum skew quadrupole settings).

TABLE I. Nominal settings for the photocathode drive-laser, $\mathrm{rf}$ gun and booster cavity during the flat-beam experiment.

\begin{tabular}{lcc}
\hline \multicolumn{1}{c}{ Parameter } & Value & Unit \\
\hline Laser injection phase & 25 & Degree \\
rms laser spot size on cathode & $0.75-1$ & $\mathrm{~mm}$ \\
rms laser pulse duration (Gaussian) & $\sim 3$ & $\mathrm{ps}$ \\
Bunch charge & 0.5 & $\mathrm{nC}$ \\
Accelerating gradient on cathode & 32 & $\mathrm{MV} / \mathrm{m}$ \\
Axial magnetic field on cathode & $400-900$ & $\mathrm{Gauss}$ \\
Booster-cavity peak electric field & 23 & $\mathrm{MV} / \mathrm{m}$ \\
\hline \hline
\end{tabular}

\section{EXPERIMENTAL AND NUMERICAL RESULTS}

Given the experimental conditions, numerical simulations are performed with the tracking program ASTRA [27]. Using the simulation outputs of the beam properties at the entrance of the transformer, the aforementioned simplex minimization algorithm is used to determine the skew quadrupole settings needed to transform the magnetized round beam into a flat beam. In the experiment, the quadrupole settings are then empirically fine tuned to insure the $x-y$ correlation on the beam has been removed downstream of the transformer. This is achieved by observing the beam transverse image on the viewers downstream of the transformer: upon removal of the angular momentum, the beam should remain flat and upright; see Fig. 3. In Table II we compare, for two cases of rms drive-laser spot sizes $\left(\sigma_{c}=\right.$ $0.76 \mathrm{~mm}$ and $\sigma_{c}=0.97 \mathrm{~mm}$ ), the final quadrupole cur- 

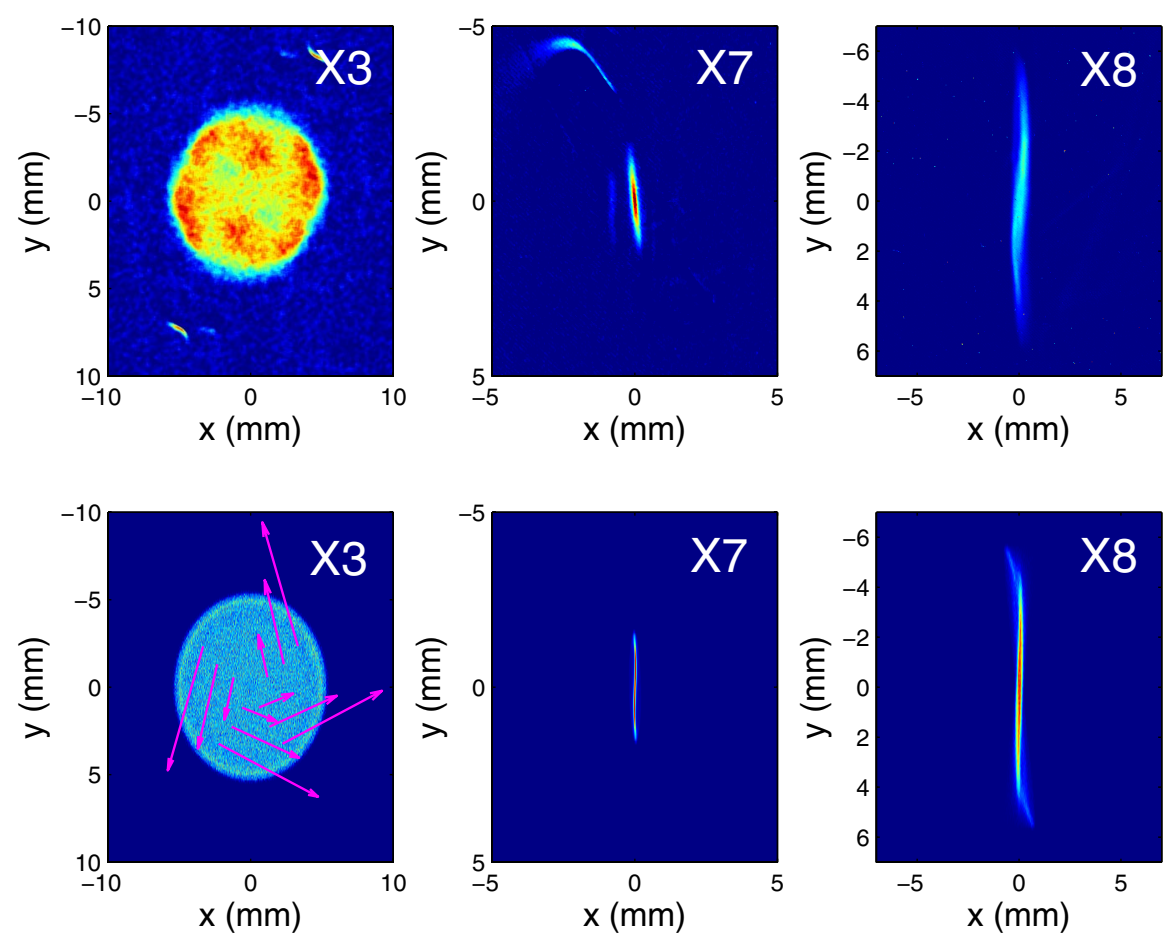

FIG. 3. (Color) Example of the transformation of the incoming angular-momentum-dominated round beam (measured at X3 viewer) into a flat beam. The beam transverse distribution is highly asymmetric and remains upright on viewers X7 and X8. Top row: measurements; bottom row: corresponding numerical simulations. The arrows on bottom left plot indicate the directions of momentum computed for a subset of macroparticles. The momentum at X7 and X8 is mainly directed along the $y$ axis (not shown for clarity). [The data presented in this figure correspond to the case of $\sigma_{c}=0.97 \mathrm{~mm}$ (see text for details).]

rents used in the experiment with the initial values obtained numerically. Most of the quadrupole currents agree with predicted values, the larger discrepancies observed for the settings of the last quadrupole is due to the looser tolerance on this quadrupole setting [21]. For the transverse emittance measurements, the beam images on the different viewers are recorded for a single-bunch beam. In Fig. 4, we present the set of experimental images, along with their respective simulated images, needed to infer the two transverse flat-beam emittances. Several shots of each of the particular images are taken and analyzed to obtain the rms beam sizes. The results are averaged and a statistical error is attributed to the mean beam sizes. The rms beam sizes, estimated on $95 \%$ of the total integrated image intensity, are then used to compute the normalized transverse emittance following the method described in Sec. II. The systematic errors on the emittance values are estimated from error propagation given the uncertainties on the beam size measurement (mainly due to uncertainties on the calibration of the imaging system). In Table III, we gather the measured and simulated parameters for the case of $\sigma_{c}=0.97 \mathrm{~mm}$. The smaller of the flat-beam emittance is $\varepsilon_{n}^{x}=0.41 \pm 0.06 \mu \mathrm{m}$, a factor $\sim 1.5$ higher than the value expected from numerical simulations. To further understand this discrepancy we have estimated the impact of spurious dispersion introduced by steering magnets needed to keep the beam orbit close to the beam line axis. The steering magnets settings used during the flatbeam experiments were loaded in the program ELEGANT [28] in order to estimate the dispersion functions along the beam line. Given the beam's rms fractional momentum spread value $\sigma_{\delta} \simeq 2.5 \times 10^{-3}$, the resulting beam sizes, i.e., including the contribution from spurious dispersion, are shown in Fig. 5. At the location of viewer X7, used for

TABLE II. Comparison of the experimental skew quadrupole currents with the numerical predictions for different laser spot sizes $\sigma_{c}$. $I_{i}$ is the current of the skew quadrupole $S_{i}$.

\begin{tabular}{lcccc}
\hline \hline & \multicolumn{2}{c}{$\sigma_{c}=0.79 \mathrm{~mm}$} & \multicolumn{2}{c}{$\sigma_{c}=0.97 \mathrm{~mm}$} \\
Quadrupole current & Experiment & Simulation & Experiment & Simulation \\
\hline$I_{1}(\mathrm{~A})$ & -1.92 & -2.03 & -1.97 & -1.98 \\
$I_{2}(\mathrm{~A})$ & 2.40 & 2.57 & 2.56 & 2.58 \\
$I_{3}(\mathrm{~A})$ & -2.99 & -4.01 & -4.55 & -5.08 \\
\hline \hline
\end{tabular}



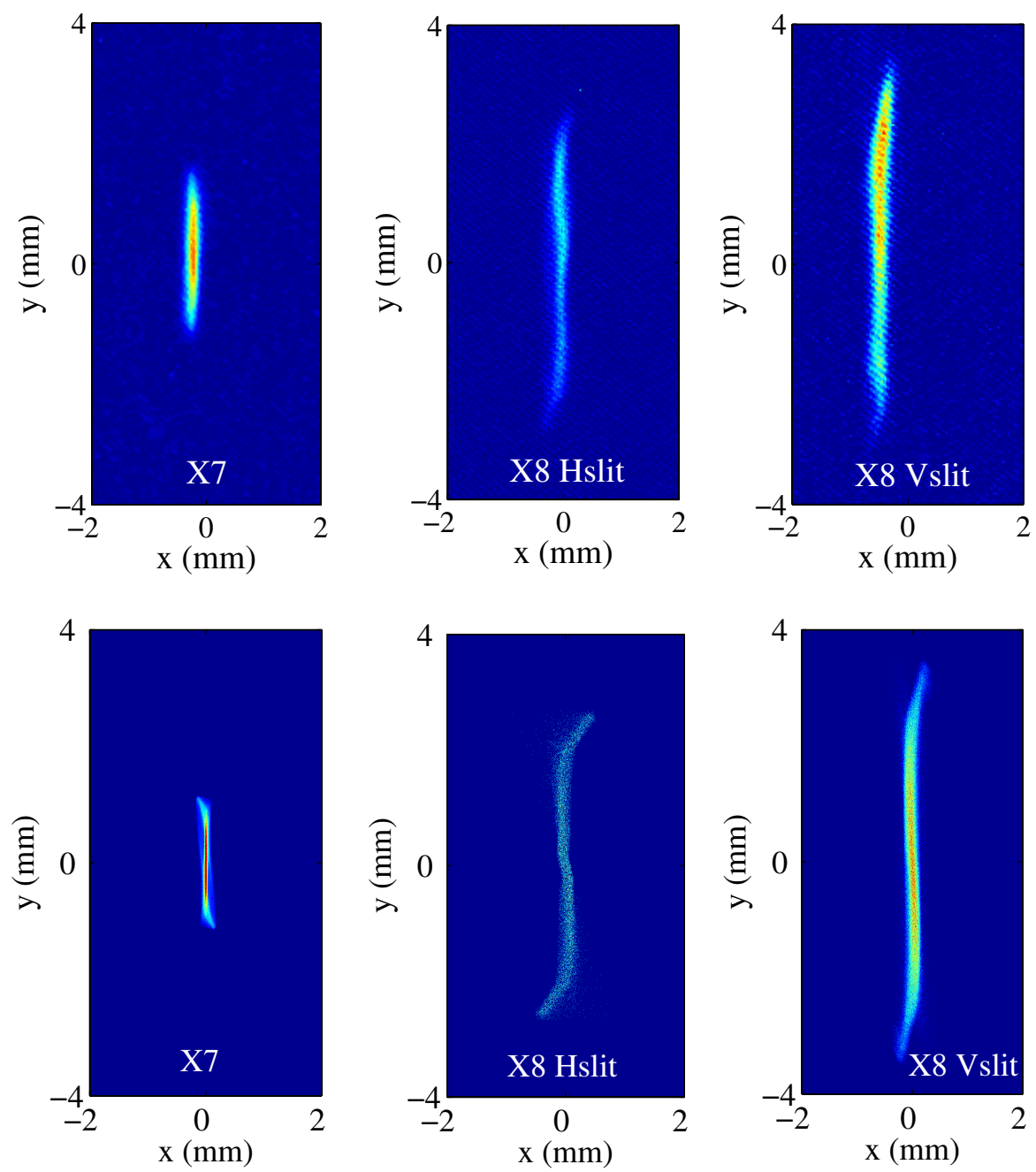

FIG. 4. (Color) The top three images are taken with digital cameras: beam at X7, horizontal and vertical slit images at X8. The bottom three are the corresponding beam profiles from ASTRA simulations. These images are associated with the flat-beam data presented in Table III.

the emittance measurement, the horizontal rms beam size increases from $58 \mu \mathrm{m}$ to approximately $95 \mu \mathrm{m}$ due to spurious dispersion. This latter value is consistent with the measurement reported in Table III thereby confirming the impact of spurious dispersion on the smaller of the flatbeam emittance measurement. Further error analysis in-

TABLE III. Measured and simulated flat-beam parameters for $\sigma_{c}=0.97 \mathrm{~mm}$. Both systematic and statistical (in brackets) error bars are included.

\begin{tabular}{lccc}
\hline \hline Parameter & Experiment & Simulation & Unit \\
\hline$\sigma_{x}^{X 7}$ & $0.088 \pm 0.01( \pm 0.01)$ & 0.058 & $\mathrm{~mm}$ \\
$\sigma_{y}^{X 7}$ & $0.63 \pm 0.01( \pm 0.01)$ & 0.77 & $\mathrm{~mm}$ \\
$\sigma_{x}^{X 8, v}$ & $0.12 \pm 0.01( \pm 0.01)$ & 0.11 & $\mathrm{~mm}$ \\
$\sigma_{y}^{X 8, h}$ & $1.68 \pm 0.09( \pm 0.01)$ & 1.50 & $\mathrm{~mm}$ \\
$\varepsilon_{n}^{x}$ & $0.41 \pm 0.06( \pm 0.02)$ & 0.27 & $\mu \mathrm{m}$ \\
$\varepsilon_{n}^{y}$ & $41.1 \pm 2.5( \pm 0.54)$ & 53 & $\mu \mathrm{m}$ \\
$\varepsilon_{n}^{y} / \varepsilon_{n}^{x}$ & $100.2 \pm 20.2( \pm 5.2)$ & 196 & \\
\hline \hline
\end{tabular}

cluding possible error on the quadrupole settings were performed via Monte Carlo technique using ELEGANT . The results are summarized in Fig. 6; and we observe a very small variation of the larger flat-beam emittance.

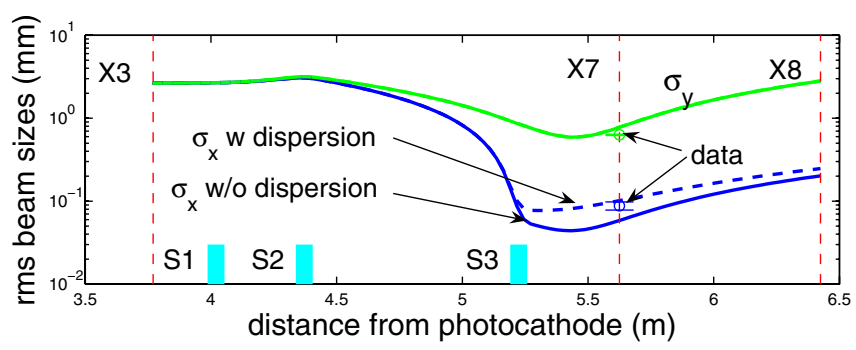

FIG. 5. (Color) Evolution of the transverse beam rms sizes along the transformer with (solid lines) and without (dash lines) the contribution of spurious dispersion. The circles correspond to the horizontal (blue) and vertical (green) measured beam sizes (see Table III). The vertical dash lines and the filled boxes, respectively, represent the viewer and skew quadrupole locations. 

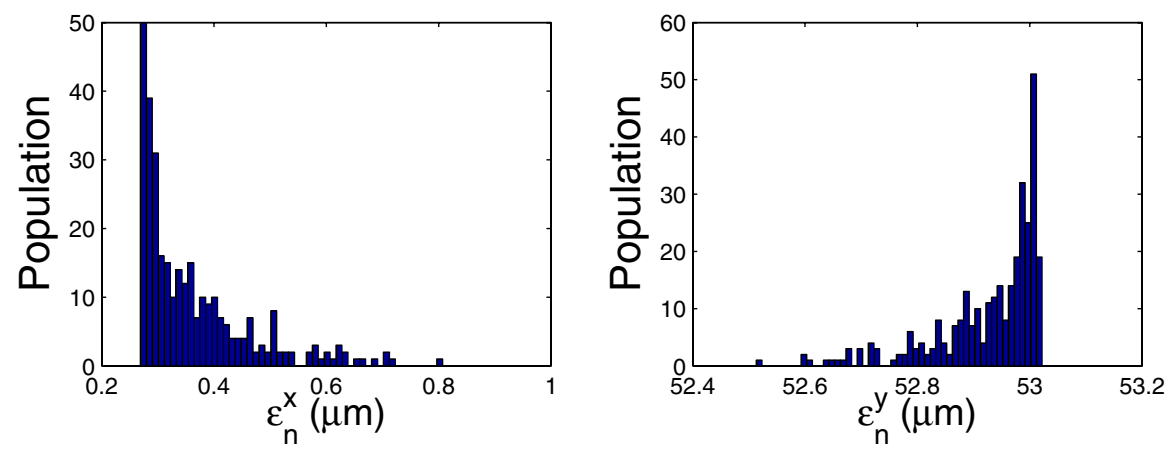

FIG. 6. (Color) Emittances variations due to nonoptimum quadrupole settings. The quadrupole settings were randomly varied from their nominal value by $2 \%$ (rms). Only simulations results corresponding to settings that provide a beam with an upright spot size at X7 and X8 were retained in the histograms (in order to mimic the experimental method used to tune the skew quadrupoles). The right and left plots correspond, respectively, to the horizontal and vertical flat-beam emittances.

The smaller of the flat-beam emittance is less than half of the expected thermal emittance due to the photoemission process of the cesium telluride material. From [29,30], we infer the thermal emittance to be $\varepsilon_{\text {th }}=0.99 \pm$ $0.10 \mu \mathrm{m}$ given $\sigma_{c}=0.97 \pm 0.05 \mathrm{~mm}$.

To gain more insight into the round-to-flat-beam transformation, we compare the expected flat-beam emittances, $\varepsilon_{n}^{ \pm}$in Eq. (1), given the incoming magnetized beam parameters, with the measured flat-beam emittances downstream of the transformer. The uncorrelated emittance of the magnetized beam $\varepsilon_{n}^{u}$ is measured using the slit technique from the beam image at X3 and the corresponding slit images at X5. $\mathcal{L}$ has been obtained with the two different methods detailed in [17]. The resulting measurements for the case $\sigma_{c}=0.97 \mathrm{~mm}$ are summarized in Table IV: within the experimental errors we observed that the measured four-dimensional (4D) emittance $\varepsilon_{4 D} \equiv \sqrt{\varepsilon_{n}^{x} \varepsilon_{n}^{y}}$ is conserved during the round-to-flat-beam transformation. We note, for this particular measurement, a $\sim 25 \%$ discrepancy for the measured larger flat-beam emittance compared to the simulation and the value predicted from the round-beam parameters. We attribute this discrepancy to a measurement error for this particular point (generally the larger flat-beam emittance agrees well with simulation; see Fig. 7). We finally report the dependence of $\varepsilon_{n}^{+}$versus $\mathcal{L}$. The value of $\mathcal{L}$ was varied either by changing $B_{0}$ or $\sigma_{c}$. As expected $\varepsilon_{n}^{+}$depend linearly on $\mathcal{L}$, and a linear regression

TABLE IV. Parameters measured from the angular-momentum-dominated round beam and the corresponding flat beam.

\begin{tabular}{lccc}
\hline \hline Parameters & Round beam & Flat beam & Simulation \\
\hline$\beta \gamma \mathcal{L}$ & $25.6 \pm 2.6$ & & 26.3 \\
$\varepsilon_{n}^{u}$ & $5.1 \pm 0.9$ & & 3.8 \\
$\varepsilon_{n}^{+}$ & $53.8 \pm 5.4^{\mathrm{a}}$ & $41.0 \pm 2.5$ & 53 \\
$\varepsilon_{n}^{-}$ & $0.49 \pm 0.22^{\mathrm{a}}$ & $0.41 \pm 0.06$ & 0.27 \\
$\sqrt{\varepsilon_{n}^{+} \varepsilon_{n}^{-}}$ & $5.1 \pm 0.9$ & $4.1 \pm 0.8$ & 3.8 \\
\hline \hline
\end{tabular}

${ }^{\mathrm{a}}$ Expected value given the measured round-beam parameters. gives $\varepsilon_{n}^{+}=(1.78 \pm 0.26) \mathcal{L}$; see Fig. 7 . The slope is in agreement with the theoretically expected slope value of 2 in the limit $\mathcal{L} \gg \beta \gamma \varepsilon_{n}^{u}$; see Eq. (1).

\section{CONCLUSION}

In summary we generated and characterized a highly asymmetric beam in a photoinjector. The lower limit for the best measured emittance ratio of $\sim 100$ is limited by our experimental setup: the fact that the transformation occurs at low energy along with $\sigma_{\delta} \simeq 0.25 \%$ made our measurement sensitive to spurious dispersion. Simulations based on steering dipole settings used to correct the beam orbit indicate that the thereby introduced dispersion could result in an overestimation of the smaller flat-beam emittance by a factor up to 2. Spurious dispersion accounts for most of the discrepancy between numerical simulations and mea-

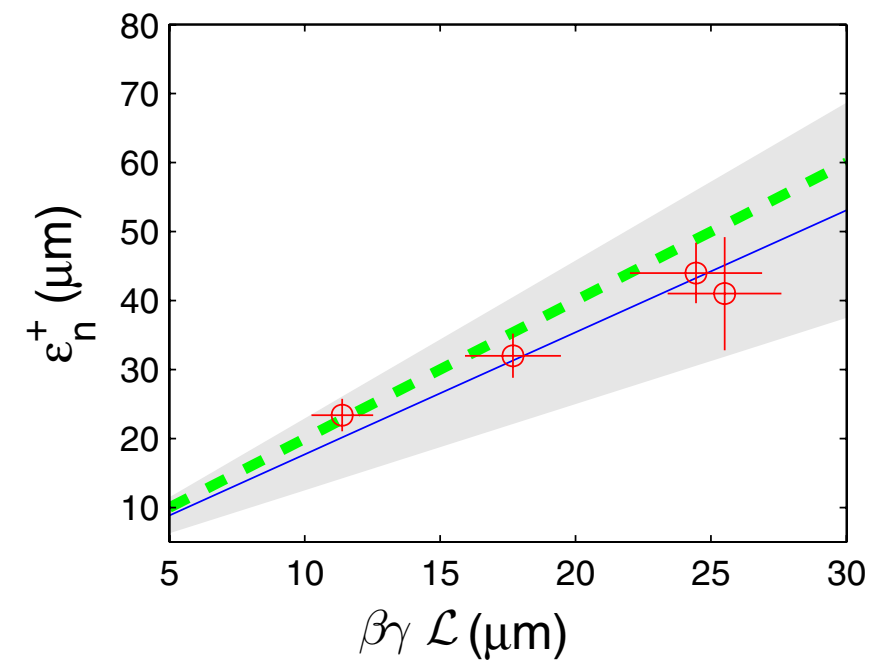

FIG. 7. (Color) Larger one of the flat-beam emittances $\left(\varepsilon_{n}^{+}\right)$ versus $\beta \gamma \mathcal{L}$. A linear regression (solid line) of the experimental data (circle) is compared with the theoretical dependence (dash line). The shaded area represents the $95 \%$ confidence bounds associated with the linear regression. 
surements. The experiment is limited to low charge in order to avoid space charge to significantly impact the beam dynamics in the transformer at $16 \mathrm{MeV}$. These two deleterious effects (dispersion and space charge) could be greatly suppressed by performing a flat-beam experiment with an higher energy electron beam.

Nonetheless our measurements support the potential flat-beam injector designs either for a proposed light source such as LUX or envisioned terahertz radiation sources based on Smith-Purcell effect. The flat-beam technique discussed in this paper might also have application in sheet-beam klystrons such as described in Ref. [31].

Finally our results open a possible path for the production of flat $e^{-}$beam for the ILC, where the main challenge is to also achieve, for a charge $Q=3.2 \mathrm{nC}$, a $4 \mathrm{D}$ emittance $\varepsilon_{4 D} \sim 0.4 \mu \mathrm{m}$ together with a transverse emittance ratio of $\sim 300$. The required $4 \mathrm{D}$ emittance value is 1 order of magnitude lower than what our photoinjector can presently produce at $Q=0.5 \mathrm{nC}$.

\section{ACKNOWLEDGMENTS}

We wish to acknowledge C. L. Bohn of Northern Illinois University, D. Edwards and H. Edwards of Fermilab, for their comments and fruitful discussions. We are thankful to J. Li and R. Tikhoplav of University of Rochester for their work on improving the photocathode laser. This work was supported by Universities Research Association Inc. under Contract No. DE-AC02-76CH00300 with the U.S. Department of Energy and by the Northern Illinois Center of Accelerator and Detector Development (NICADD).

[1] K. Yokoya and P. Chen, in Proceedings of the 1989 Particle Accelerator Conference, Chicago, IL, 1989, edited by F. Bennett and J. Kepta (IEEE, New York, NY, 1989), pp. 1438-1440.

[2] W. Barry et al., Lawrence Berkeley National Lab. Report No. LBNL-51766, 2002.

[3] A. Zholents, P. Heimann, M. Zolotorev, and J. Byrd, Nucl. Instrum. Methods Phys. Res., Sect. A 425, 385 (1999).

[4] S. J. Smith and E. M. Purcell, Phys. Rev. 92, 1069 (1953).

[5] K.-J. Kim and S.-B. Song, Nucl. Instrum. Methods Phys. Res., Sect. A 475, 158 (2001); V. Kumar and K.-J. Kim, Phys. Rev. E 73, 026501 (2006); C. L. Bohn, Y.-E Sun, and K.-J. Kim (unpublished); available at http://nicadd.niu.edu/presentations/BohnFNPLspeoi.doc

[6] H.L. Andrews and C.A. Brau, Phys. Rev. ST Accel. Beams 7, 070701 (2004).

[7] Y. Zhang, Ya. Derbenev, and R. Li, Nucl. Instrum. Methods Phys. Res., Sect. A 507, 459 (2003).

[8] http://lcdev.kek.jp/ILCWS/

[9] Y. Honda et al., Phys. Rev. Lett. 92, 054802 (2004).

[10] J. B. Rosenzweig, E. Colby, G. Jackson, and T. Nicol, in Proceedings of the 1993 Particle accelerator Conference,
Washington $D C$, edited by S.T. Corneliussen (IEEE, Piscataway, NJ, 1993), pp. 3021-3023; G. Fiorentini, C. Pagani, and L. Serafini, in Proceedings of the 1995 Particle Accelerator Conference, Dallas, TX, edited by L. T. Gennar and R. H. Siemann (IEEE, Piscataway, NJ, 1995), pp. 973-975.

[11] Ya. Derbenev, University of Michigan Report No. UMHE-98-04, 1998.

[12] R. Brinkmann, Y. Derbenev, and K. Flöttmann, DESY Report No. TESLA 99-09, 1999; Phys. Rev. ST Accel. Beams 4, 053501 (2001).

[13] A. Burov and V. Danilov, Report No. FERMILAB-TM2043, 1998.

[14] D. Edwards et al., in Proceedings of the XX International Linac Conference, Monterey, CA, 2000 (SLAC, Stanford, 2000), pp. 122-124.

[15] D. Edwards et al., in Proceedings of the 2001 Particle Accelerator Conference, Chicago, IL (IEEE, Piscataway, NJ, 2001), pp. 73-75.

[16] E. Thrane et al., in Proceedings of the XXI International Linac Conference, Gyeongju, Korea (Pohang Accelerator Laboratory, Pohang, Korea, 2002), pp. 308-310.

[17] Y.-E Sun et al., Phys. Rev. ST Accel. Beams 7, 123501 (2004).

[18] A. Burov, S. Nagaitsev, and Ya. Derbenev, Phys. Rev. E 66, 016503 (2002).

[19] K.-J. Kim, Phys. Rev. ST Accel. Beams 6, 104002 (2003).

[20] Y.-E Sun et al., in Proceedings of the XXII International Linac Conference (LINAC 2004), Lubeck, Germany, 2004, pp. 150-152 (proceedings available at http://accelconf.web.cern.ch/accelconf/104/INDEX.HTML).

[21] Y.-E Sun, K.-J. Kim, and P. Piot, in Proceedings of the Particle Accelerator Conference, Knoxville, TN, 2005 (IEEE, Piscataway, NJ, 2005); see also Report No. fermilab-conf-05-158-AD, 2005.

[22] http://nicadd.niu.edu/fnpl

[23] B. Aune et al., Phys. Rev. ST Accel. Beams 3, 092001 (2000).

[24] C. L. Bohn (private communication).

[25] Y.-E Sun, Ph.D. thesis, University of Chicago [Report No. Fermilab-thesis-2005-17, 2005], available at http:// fnalpubs . fnal .gov/archive/thesis/fermilab-thesis - 2005 17.shtml

[26] C. Lejeune and J. Aubert, Adv. Electron. Electron Phys. Suppl. A 13, 159 (1980).

[27] K. Flöttmann, ASTRA: A Space Charge Tracking Algorithm, http://www.desy.de/ mpyflo

[28] M. Borland, Advanced Photon Source Report No. LS-287, 2000.

[29] V. V. Miltchev et al., in Proceedings of the 26th FreeElectron Lasers Conference, Triestre, Italy, 2004 (Comitato Conferenze Elettra, Trieste, Italy, 2004), pp. 399-402.

[30] K. Flöttmann, DESY Report No. TESLA-FEL-1997-01, 1997.

[31] S. J. Russell et al., Phys. Rev. ST Accel. Beams 8, 080401 (2005). 